\title{
Design and Manufacture of Optimized Continuous Composite Fiber Filament Using Additive Manufacturing Systems
}

De Vivo Luca*

Department of Structural Engineering, University of California, San Diego, California, USA

\begin{abstract}
The main purpose of this study is to investigate the current state of Additive Manufacturing of Fiber Reinforced Plastics technology. Recent advances in additive manufacturing, with the introduction of fused deposition modeling of continuous fiber-reinforced thermoplastics, have opened a door for further improvements in composite manufacturing. We explore in detail the different available methods of manufacturing structures with continuous fiber reinforced plastics using additive manufacturing. It was found that it's possible to print different types of fibers; Glass, Carbon, Kevlar and Yute (Bio Filaments), and there are two types of resins that are mainly used; thermosets and thermoplastics. Finally, we conclude by analyzing current challenges of this technology in the area of design and structural optimization.
\end{abstract}

Keywords: Filament; Additive manufacturing; Stereolithography

\section{Introduction}

Additive Manufacturing (AM) is a process where machines automatically fabricate arbitrary shaped parts, pixel by pixel, layer by layer, from almost any material, as opposed to subtractive manufacturing techniques. AM has many strategic advantages over traditional manufacturing processes: the production of complex internal and external part geometries that cannot be made in any other way; rapid iteration through design permutations; and the ability to build customized functional parts in small lot sizes to meet end-user's specifications [1]. The technology first emerged in 1987 with stereolithography (SL) [2], and since then has evolved from a limited number of expensive prototypes to widely available, small-scale commodity production tools. AM is expected to transform every aspects of our lives, and some people are calling it "the second industrial revolution". There are many different additive manufacturing processes, all with their specific benefits, drawbacks and application areas. Currently the available AM processes are divided into 7 groups [3]. Material Extrusion, Material Jetting, Binder Jetting, Sheet Lamination, V at Photo-polymerization, Powder Bed Fusion, and Direct Energy Deposition.

\section{Literature Review}

The materials required for the final product will determine what AM process is used. Polymers (Figure 1) and metals are the most common types of materials used in AM and can be used to produce molds, prototypes and functioning components [4]. There are however possibilities for other materials to be used in AM, such as Medical and Biomedical graded materials, glass, and other ceramics, advancedfibers and food. Additive Manufacturing has distinct advantages and disadvantages, and it's up to the designer or engineer to decide if additive manufacturing is the best fit for the project. Some of the main advantages of AM are: variety is free, reduced lead time, variety if free, little lead time, and little skill manufacturing, few constraints, and less waste. On the other hand major disadvantages related to AM are: slow build rates, higher production costs, requires post processing, and poor mechanical properties in certain directions.

Even though AM has greatly evolved over the past three decades it still has a lot of room for improvements. One of the areas that have not yet been deeply investigated is the combination of composite techniques with additive manufacturing. In recent years the use of composite laminated plates have been widely adopted by the engineering community especially in practices such as aerospace, marine, civil and medical engineering. Composite materials are made by combining two or more materials, which often have different properties, in order to create a material with unique properties. One of the most widely used composite materials is fiber-reinforced composite (FRC), where fibers and a matrix are used to create structures with an exceptional strength to weight ratio [5]. The fiber provides strength in the longitudinal direction while the matrix, often a resin, provides stiffness and bonding of the fibers. A special characteristic of FRC is that multiple plies with different fiber orientations can be stacked in order to provide strength in multiple directions (Figure 2), which allows composite structuctures to be tailor for specific applications or load cases.

As mentioned earlier polymers constitute one of the most popular AM materials, yielding lightweight but inherently weak components that cannot hold up against high tension and bending stresses. A need for improved tensile strength has driven a recent interest in AM of fiber reinforced polymers (FRPs). AM-FRPs are separated into two different families; short fiber reinforcement [6], and continuous long fiber reinforcement [7-13]. AM-FRPs reinforced with short fibers have demonstrated increased mechanical strength, but with limited design and structural flexibility. AM-FRPs reinforced with continuous fibers provide structural reinforcement within plane. We will focus our attention to previous works on AMF-FRPs of continuous fibers and the different methods of achieving composite structures using this technique.

Recent improvements in the area of additive manufacturing allows the production of complex arrangement of fibers using a technique known as Fused Deposition Modeling (FDM) of continuous fiber reinforced plastics. Automated fiber placement of thermoset

*Corresponding author: Luca De Vivo Nicoloso, Department of Structural Engineering, University of California, San Diego, California, USA, Tel: +1 858-5342230; E-mail: Idevivo@ucsd.edu

Received July 21, 2017; Accepted July 29, 2017; Published August 10, 2017

Citation: Luca DV (2017) Design and Manufacture of Optimized Continuous Composite Fiber Filament Using Additive Manufacturing Systems. J Material Sci Eng 6: 363. doi: 10.4172/2169-0022.1000363

Copyright: (c) 2017 Luca DV. This is an open-access article distributed under the terms of the Creative Commons Attribution License, which permits unrestricted use, distribution, and reproduction in any medium, provided the original author and source are credited. 


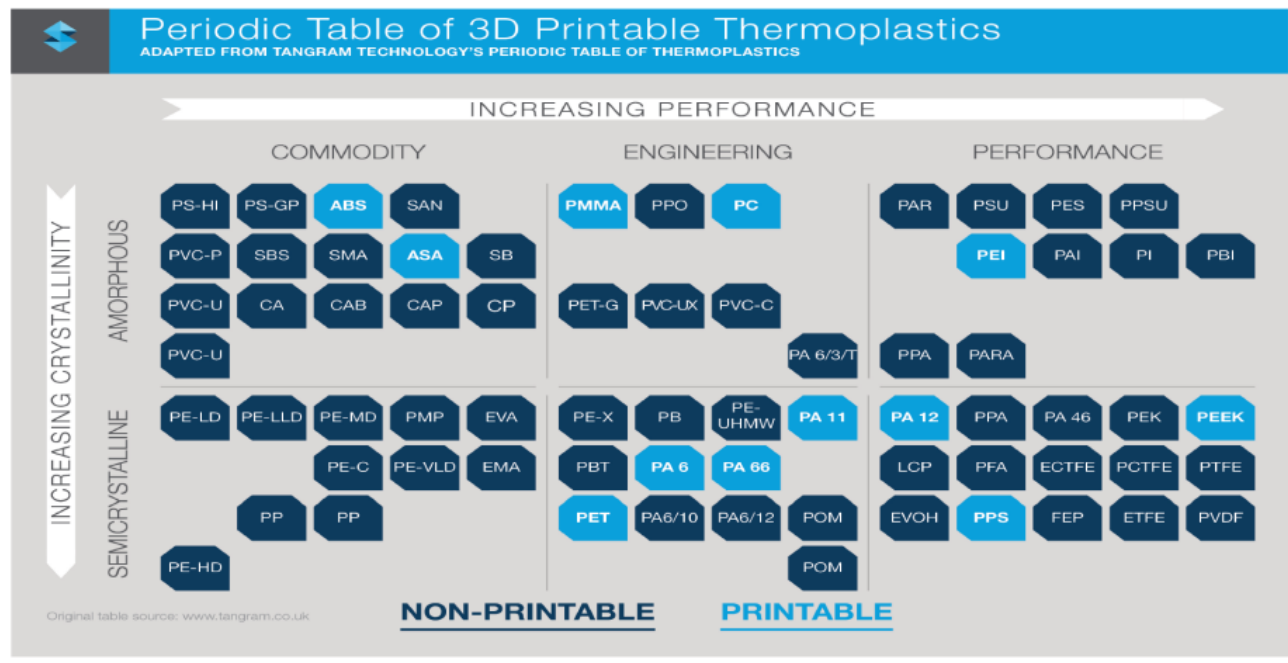

Figure 1: Stratasys periodic table of 3D printable thermoplastic polymers.
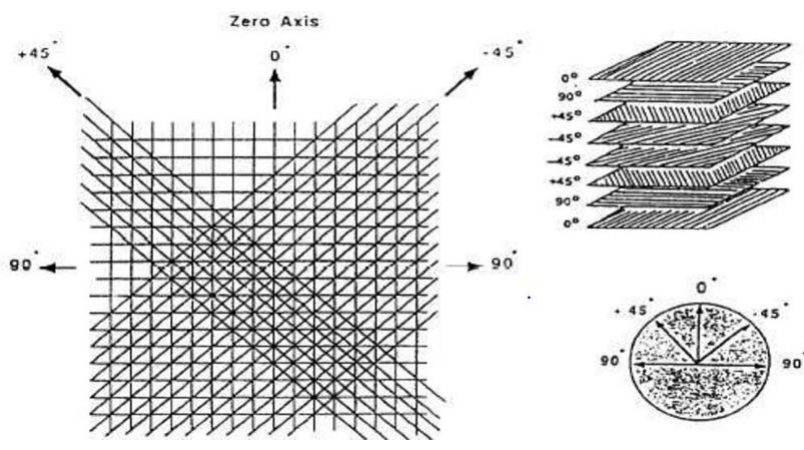

Figure 2: Standard composite ply orientation

Composites were invented over 40 years ago and is now widely accepted for the manufacture of advanced composite structures. However, expensive tooling and post process such as autoclaves are required. In-situ inexpensive tooling, eliminates these post process, and can be considered an additive manufacturing process for advanced composite structures.

Griffini, Invernizzi and Natale developed a UV-Assisted 3D printing of glass (GFR) and carbon fiber reinforced (CFR) dual-core polymer composite [11]. In this work a sequential interpenetrating polymer network (IPN) obtained by formulating a photocurable acrylic resin with a thermo-curable epoxy resin was presented and proposed as matrix for the fabrication of GFR and CFR composite structures by means of $3 \mathrm{D}$ printing technology. The resin material combines an acrylic based photocurable resin with a low temperature (140 C) thermally curable resin system based on bisphenol and diglycidyl ether as base component, it uses aliphatic anhydride as hardener and (2,4,6,-tris(dimethylaminomethyl)phenol) as catalyst. Photoinitiated polymers played a key role in the development of this experimental work. Photopolymers belong to the family of materials that change their properties when exposed to ultraviolet radiation. In order to successfully print with this new material a new 3D printer had to be developed (Figure 3). The apparatus consist of a system of rollers that feed the fiber inside the impregnation station; a head of power, which comprises a polymerization device (eg. a UV LED or laser light or a thermal emission source), positioned at the nozzle; a cutting tool for the composite material, configured to interrupt the supply of the material in the nozzle exit, and a numerical control machine of at least 3 axes that has the task of moving the head of the material supply. UV3D printed macrostructures were successfully demonstrated giving a clear indication of their potential use in real life structural applications. The 3D printed structures were characterized by good thermal stability and mechanical properties.

Another company using a similar composite UV curing technique is Functional 3D Printing $[9,10]$. They invented a machine capable of $3 \mathrm{D}$ printing continuous composite structures using a new positioning system utilizing hollow tubular printing techniques and multiple print heads working together in tandem to continuously manufacture large advanced structures. Their technology harness UV light to instantly cure a combination of continuous fiber and thermoset matrix material giving them the ability to print into free space. Rapid initiation of free radical polymerization is promoted by a precise control of high intensity irradiation to quickly promote crosslinking of the thermoset matrix among the continuous fibers. They are capable of printing with 8 and 16 channel nozzles utilizing different material combinations (Figure 4, Left). The printer is capable of printing 4 different material families (Figure 4, Right); Hollow Core Multi-Functional (Hollow core with fiber optics, carbon fiber and polymer encasement), Multi-Strand Fiber Optic Core (Fiber optic core is encapsulated by continuous metal wire, carbon fiber, and polymer encasement), Basic CC3D Material (combination of thermoset acrylate and carbon fiber), and Carbon Fiber with Metal Core.

Matsuzaki and Ueda et al. developed a method for three-dimensional (3D) printing of continuous fiber-reinforced thermoplastics by in nozzle impregnation based on FDM [7]. A thermoplastic filament and continuous fibers are separately supplied to the $3 \mathrm{D}$ printer and the fibers were impregnated with the filament within the heated nozzle of the printer immediately before printing (Figure 4). The reinforcing fibers are heated using a nichrome wire before entering the nozzle, to enhance the permeation of the fiber bundles with thermoplastic resin; the heat diffuses to the resin and decreasing the viscosity of the PLA. The reinforced filament is heated to a temperature greater than a melting temperature of the matrix material $(210 \mathrm{C})$ and less than a melting temperature of the core fiber prior to extruding the filament from the extrusion nozzle. Polylactic acid (PLA) was used as the matrix 
Citation: Luca DV (2017) Design and Manufacture of Optimized Continuous Composite Fiber Filament Using Additive Manufacturing Systems. J Material Sci Eng 6: 363. doi: 10.4172/2169-0022.1000363

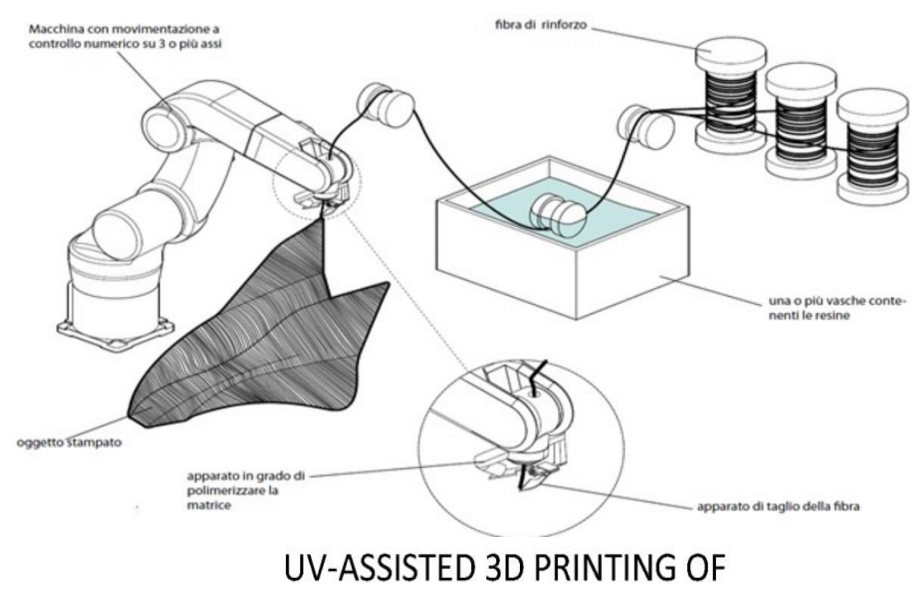

FIBER-REINFORCED DUAL-CURED POLYMER COMPOSITES

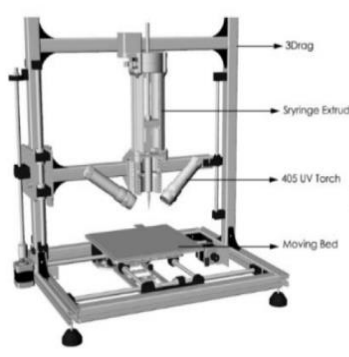

3D PRINTER

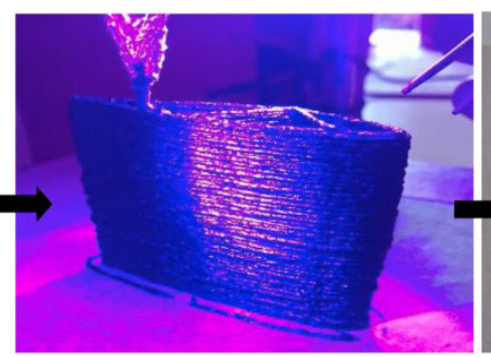

PRINTING PROCESS

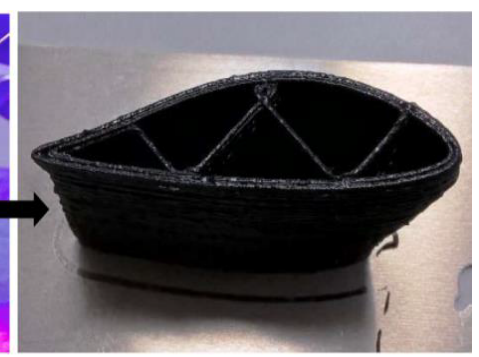

CARBON-FILLED PRINTED AIRFOIL

Figure 3: UV-assisted 3D printer of GFR and CFR.
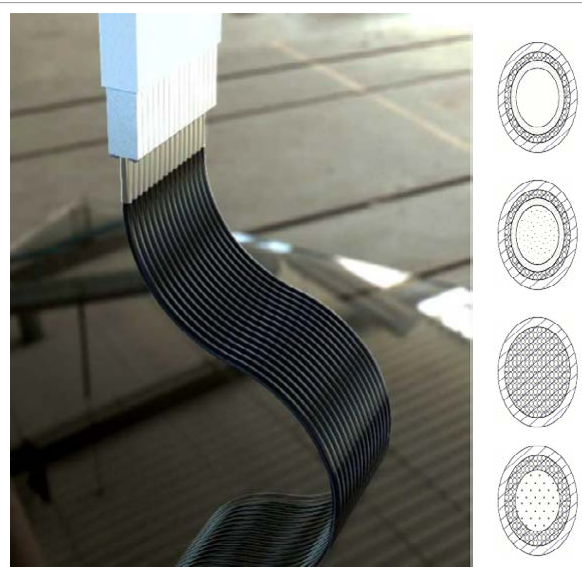

HOLLOW CORE MULTI-FUNCTIONAL

This example encompasses a hollow core with fiber optics, carbon fiber and

polymer encasement.

MULT-STRAND FIBER OPTIC CORE

Here the fiber optic core is encapsulated by continuous metal wire, carbon fiber,

and polymer encosement.

BASIC CC3D MATERIAL

Here is a basic CC3D material combination of thermoset acylate and carbon fiber

CARBON FIBER WITH METAL COPE

This example has a metal core with a carbon fiber sheath, useful for creating

conductive relinforced paths

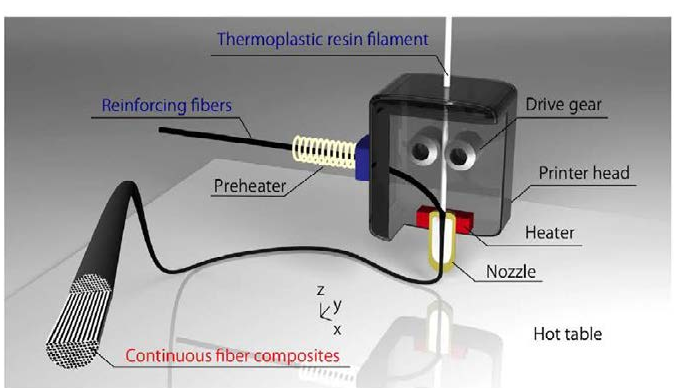

Figure 4: Left; Functional 3D printing with 16 channel nozzles. Rigth; 3D printable materials. Schematic of the 3D printer head used to produce Continuous Fiber Reinforced Thermoplastic structures using in-nozzle impregnation based on FDM. 
Citation: Luca DV (2017) Design and Manufacture of Optimized Continuous Composite Fiber Filament Using Additive Manufacturing Systems. J Material Sci Eng 6: 363. doi: 10.4172/2169-0022.1000363

while carbon or glass fibers were used as the reinforcement.The $3 \mathrm{D}$ printer for obtaining continuous fiber composites was developed by modifying the printhead of a commercially available FDM 3D printer. The technique enables direct 3D fabrication without the use of molds and may become a standard next-generation composite fabrication methodology using FDM printing. Continuous fiber reinforcement improved the tensile strength of the printed composites relative to the values shown by conventional 3D-printed polymer based composites.

Another technique proposed by MarkForged is to alternate in-plane layers of thermoplastic and fiber materials. The system has two print heads, one for plastic and one for fibers. During the printing process a layer of thermoplastic, in this case a combination of tough nylon with micro-carbon reinforcement is placed as the matrix and confinement for the continuous fibers, (Fiber, Carbon and Kevlar) which are then placed on the second layer within the plastic confinement (Figure 5, Left). This process is repeated until the final layer is printed and the piece is ready to be removed. Markforged printers are capable of producing parts that are 5-10 times stronger than conventional thermoplastic 3D printed parts (Figure 5, Right).

Turk, Triebe, and Meboldt et al. proposed to use FDM together with a multi-axis robot to place continuous fiber in three dimensions, and build structures from the inside out [9]. This was achieved by combining additive manufacturing tooling with in-situ automated fiber placement of thermoplastic composite rapid prototyping software with Automated Fiber Placement.

Another company utilizing the mobility and versatility of multi-axis robot for AM is Arevo Labs with its Robotic Additive Manufacturing Platform (RAMP) for 3D printing Complex Composite Parts [1]. RAMP consists of a robotic AM cell (Figure 6), ultra strong composite material and a comprehensive CAM software suite. The robotic AM cell employs a standard commercially available six-axis robotic arm and a composite deposition end-effector with advanced thermal management technology for processing high-performance carbon fiber reinforced thermoplastic. The Ultra Strong continuous carbon fiber reinforcement thermoplastic composite is more than five times stronger than titanium, tolerates extreme temperatures and is highly chemical resistant. And the software converts CAD models to six degrees of freedom additive manufacturing G-Code, which combined with a precise kinematics simulator interprets deposition instructions to validate and optimize part construction.

\section{Conclusion}

Over the last years we have seen incredible technological advances on the Additive manufacturing of Fiber Reinforced Plastics. These new manufacturing methods now allow composite parts to be manufactured using continuous fiber reinforcement which leads to stronger, lighter and tougher structures. Although great advance have been achieved, there are still question that remains seemingly unanswered especially in the design and analysis of these composite structures. From composite analysis we know that optimal orientation of fibers can improve structural stiffness, strength, ultimate failure load, buckling stress, and fundamental frequency of composite laminates. With the gained ability of placing fibers in any direction using the manufacturing techniques
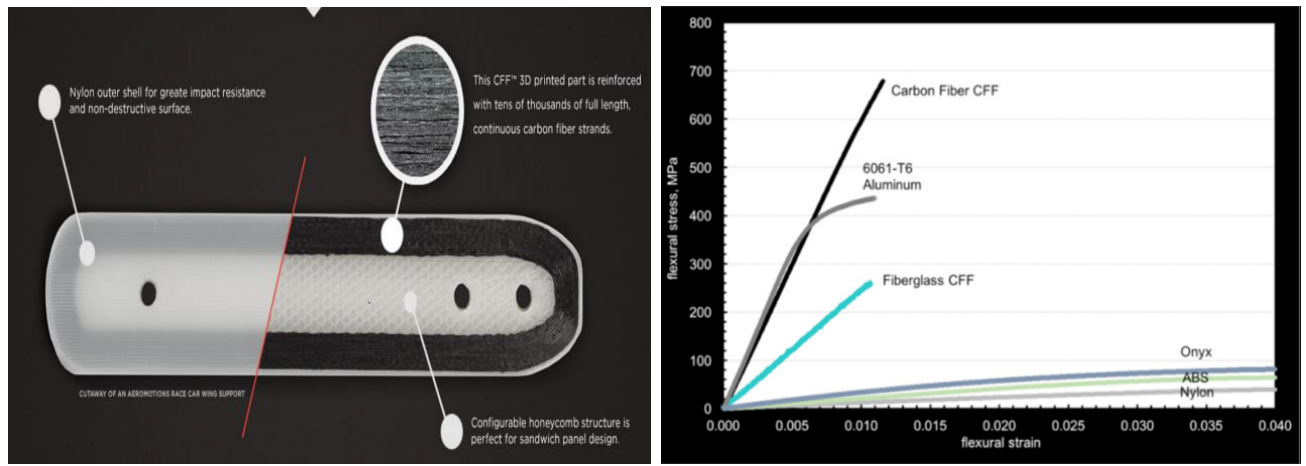

Figure 5: MarkForged Carbon Fiber printed 3D part.

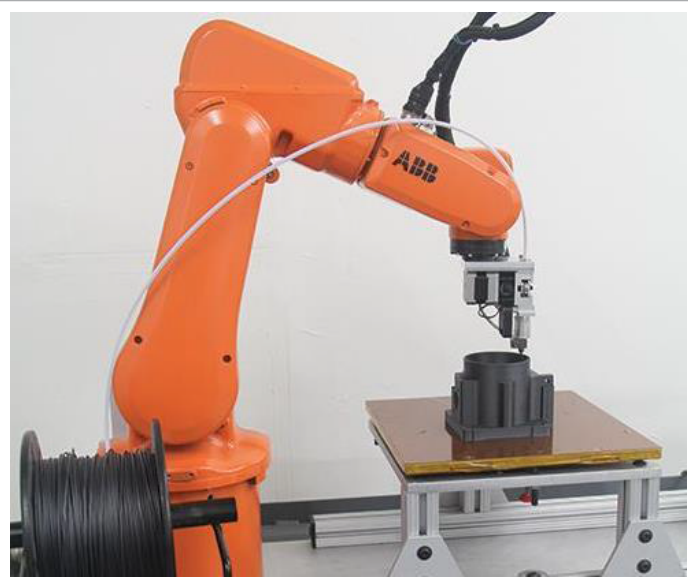

Figure 6: Arevo's RAMP 
Citation: Luca DV (2017) Design and Manufacture of Optimized Continuous Composite Fiber Filament Using Additive Manufacturing Systems. J Material Sci Eng 6: 363. doi: 10.4172/2169-0022.1000363

Page 5 of 5

mentioned above a new challenge arises, and it is the development of optimization design and slicing software. The new generation of design and slicing software would need to not only slice the CAD file into layers for the AM to take place but it will also have the task of orienting the continuous fibers in an optimal direction to yield the desired performance. This new task leads to a different challenge and it is how do we analyze and optimize structures using this new materials. A set of constitutive models will need to be developed in order to mathematically describe the mechanical behavior of structures manufactured using Additive Manufacturing-Fiber Reinforced Plastics. A design method which optimizes fiber arrangement will allow designers to take full advantage of this manufacturing technology and we believe that stronger, lighter and tougher structures could be made if implemented correctly.

\section{References}

1. Lipson $\mathrm{H}$ (2011) The shape of things to come: frontiers in additive manufacturing Frontiers of Engineering, pp: 33-44.

2. Wohlers T, Gornet T (2011) History of additive manufacturing. Wohlers Report: Additive Manufacturing and 3D Printing State of the Industry Annual Worldwide Progress Report.

3. Design, Production \& Management Research (2017) University of Twente. Additive Manufacturing Processes.
4. SPI Lasers Company (2017) Additive Manufacturing Materials.

5. Kosmatka J "Design of Composite Structures". Version 1.0. Department of Structural Engineering, University of California, San Diego.

6. Tekinalp HL, Kunc V, Velez-Garcia GM, Duty CE, Love LJ, et al. (2014) Highly oriented carbon fiber-polymer composites via additive manufacturing. Composites Science and Technology 105: 144-150.

7. Matsuzaki R, Ueda M, Namiki M, Jeong TK, Asahara H, et al. (2016) Threedimensional printing of continuous-fiber composites by in-nozzle impregnation. Scientific reports 6: 23058

8. Tse LYL, Kapila S, Barton K (2016) Contoured 3D Printing of Fiber Reinforced Polymers.

9. Tyler K (2016) US Patent No. 9,511,543. Washington, DC: U.S. Patent and Trademark Office.

10. Tyler K (2015) Method and Apparatus for Additive Mechanical Growth of Tubular Structures." U.S. Patent Application 14/810,437.

11. Invernizzi M, Natale G, Levi M, Turri S, Griffini G (2016) UV-assisted 3D printing of glass and carbon fiber-reinforced dual-cure polymer composites. Materials 9: 583.

12. Mark GT, Gozdz AS (2015) U.S. Patent No. 9,156,205. Washington, DC: U.S Patent and Trademark Office.

13. Türk DA, Triebe L, Meboldt M (2016) Combining additive manufacturing with advanced composites for highly integrated robotic structures. Procedia CIRP 50: $402-407$. 